\title{
ESTUDO DA INVERSÃO CATASTRÓFICA EM EMULSÕES DE PETRÓLEO-SISTEMA MODELO
}

\author{
Jéssica Jakubiak Bento ${ }^{2}$, Marcelle Guth ${ }^{1}$, Regina Weinschutz ${ }^{1}$, Carlos Itsuo Yamamoto ${ }^{2}$, \\ Luiz Fernando de Lima Luz Junior ${ }^{1}$ \\ ${ }^{1}$ Universidade Federal do Paraná, Departamento de Engenharia Química \\ ${ }^{2}$ Universidade Federal do Paraná, Programa Pós Graduação em Engenharia Química \\ Email para contato: jeljak@hotmail.com
}

\begin{abstract}
RESUMO - O crescimento da produção de petróleo e as descobertas de poços com óleo pesado se tornaram um desafio às refinarias, já que a viscosidade destes óleos é muito superior àqueles para os quais as plantas foram projetadas. Em meio natural, petróleo e água encontram-se geralmente em duas fases separadas. No entanto, ao realizar-se a extração em poços de petróleo ocorre a formação de emulsões água em óleo (A/O). Estas têm alta viscosidade, aumentando a perda de carga no escoamento, reduzindo a produção e aumentando os custos de produção. Diante disto, inúmeras pesquisas têm sido realizadas para solucionar este quadro e uma alternativa é a inversão das emulsões dos petróleos, transformando-as em emulsões óleo em água, O/A, reduzindo assim a viscosidade. No presente estudo verificou-se a possibilidade de inversão destas emulsões com adição de surfactantes, tanto lipofílicos quanto hidrofílicos, Span 60 e Tween 80, respectivamente. A fase oleosa é uma mistura de n-heptano e tolueno, e a fase aquosa água destilada. Para obtenção das emulsões água/óleo utilizou-se um homogeineizador a $10000 \mathrm{rpm}$, com a adoção de três diferentes métodos, sendo um deles de emulsificação direta e os outros dois de emulsificação dinâmica. A inversão realizada é do tipo catastrófica, verificada através da medida de condutividade. A análise de viscosidade mostrou redução desta de até três ordens de grandeza. Além disto, para visualizar os efeitos da inversão foi realizada microscopia das amostras.
\end{abstract}

\section{INTRODUÇÃO}

O petróleo é constituído, basicamente, por uma mistura de compostos químicos orgânicos e contém centenas de compostos químicos (Thomas, 2004). Os óleos obtidos de 
diferentes reservatórios de petróleo possuem características diferentes. Alguns são pretos, densos, viscosos, liberando pouco ou nenhum gás, enquanto que outros são castanhos ou bastante claros, com baixa viscosidade e densidade, liberando quantidade apreciável de gás. Entretanto, todos eles produzem análises elementares semelhantes, tais como: $11-14 \%$ de Hidrogênio, 83-87\% de Carbono, 0,06-8\% de Enxofre, 0,11-1,7\% de nitrogênio, 0,1-2\% de Oxigênio, e até 0,3\% de Metais (Thomas, 2004).

As emulsões são muito comuns, existem desde o processo de perfuração do poço de petróleo até a distribuição de seus derivados. Isto se deve a sua natureza oleosa e à sua composição. Define-se emulsão como uma mistura heterogênea de dois ou mais líquidos, onde um dos líquidos se dispersa em forma de gotículas em outro. Esta dispersão depende da presença de um agente emulsificante e de energia.

Essas podem ser classificadas em óleo em água (O/A), onde o óleo é a fase dispersa e a água é a fase contínua, e água em óleo $(\mathrm{A} / \mathrm{O})$ onde a água é a fase dispersa e o óleo é a fase contínua. $\mathrm{O}$ mais comum é o tipo $\mathrm{A} / \mathrm{O}$, devido à natureza hidrofóbica dos agentes estabilizantes presentes no petróleo, estas são ilustradas na Figura 1. Esta interface água-óleo acompanha partículas inorgânicas e orgânicas que constroem um filme bastante estável e mecanicamente forte (Schramm, 1992). Esta estabilidade da emulsão aumenta a viscosidade, aumentando também a perda de carga de sistema de transportes de petróleo, consequentemente diminuindo a produção dos poços e aumentando os custos para produção.

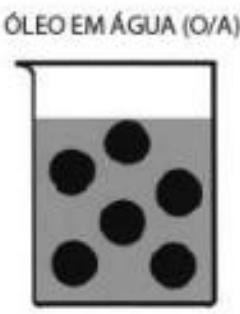

AGUA EM OLEO EM AGUA (A/O/A)

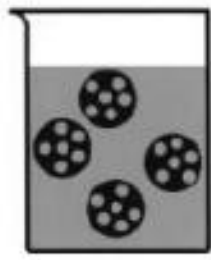

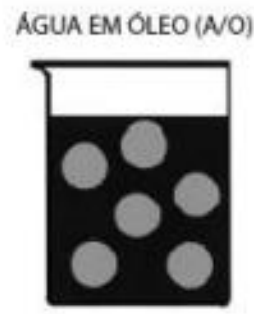

OLEO EM ÁGUA EM OLEO (O/A/O)

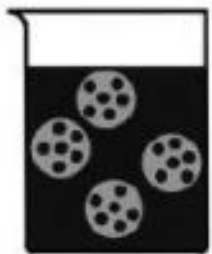

Figura 1 - Tipos de Emulsões.

Fonte: Adaptado de Schramm,1992.

O tipo da emulsão é determinado pela medida de condutividade, pois na maioria dos casos é na fase aquosa que estão presentes eletrólitos, que conduzem eletricidade, o que não ocorre na fase óleo. Além disso, o monitoramento constante da condutividade elétrica permite a determinação da inversão da emulsão (Souza, 2009). 
A viscosidade também é uma propriedade importante. E esta é diretamente proporcional à viscosidade da fase externa, que no caso da inversão de emulsão é da fase aquosa (Salager et al., 2001).

O comportamento reológico de uma emulsão pode ser Newtoniano ou não Newtoniano dependendo da composição. Em valores de concentração de fase dispersa baixos até moderados, as emulsões exibem comportamento Newtoniano. Já na faixa de altas concentrações, a emulsões possuem comportamento não Newtoniano (Schramm,1992).

Os agentes emulsificantes são componentes de superfície ativa, que se ligam à superfície da gota de água e diminuem a tensão interfacial óleo/água. Quando a energia é adicionada à mistura por agitação, as gotas da fase dispersa são quebradas em pequenas gotas (Bradley, 2001). Estas moléculas apresentam uma parte com característica apolar ligada a uma outra parte com característica polar e são chamadas de tensoativos ou surfactantes.

O uso de emulsões O/A para reduzir a viscosidade de óleos pesados permite o transporte em linhas convencionais, sem a utilização de aquecimento do óleo em bancos de trocadores de calor ao longo da linha. Os benefícios dessas emulsões podem ser aplicados no transporte do óleo e no aumento de taxas de produção de óleo cru. A redução na viscosidade pela emulsão O/A pode chegar a mais de duas ordens de grandeza (Schramm, 1992).

Para inversão de emulsões conhecem-se dois métodos, o transicional e o catastrófico. A inversão catastrófica ocorre quando uma emulsão muda de O/A para A/Oou vice-versa devido a uma adição excessiva da fase interna. A fase adicionada é primeiramente dispersa em pequenas gotas, a quantidade de gotas aumenta até o ponto que ocorre a inversão. Esta reação pode acontecer rapidamente ou passar por uma evolução contínua (Tyrode et al., 2003).

Por razões econômicas é desejável transportar mais óleo e a menor quantidade de água possível. Para manter a viscosidade da emulsão abaixo do requerido nas especificações das linhas de transporte (tipicamente por volta de $400 \mathrm{cP}$ na temperatura ambiente), há um valor máximo de $70 \%$ a $75 \%$ de óleo pesado, pois em valores maiores a viscosidade se torna muito alta, pois com o aumento da fase interna a dissipação da energia de cisalhamento se torna maior. Utilizar emulsões O/A para reduzir a viscosidade está sendo avaliada como uma alternativa para o uso de diluentes ou calor, para facilitar o escoamento. Além disso, as emulsões inversas são menos suscetíveis à temperatura que as emulsões A/O. Outro fator importante é que ao partir o sistema de bombeamento da linha, após uma parada de emergência, a reemulsificação do óleo não gera nenhum problema (Langevin et al., 2004).

\subsection{Objetivo}

Estudar a inversão de emulsões, avaliando a influência da composição do óleo, o surfactante utilizado e a temperatura, a fim de se obter um baixo ponto de inversão e redução da viscosidade da emulsão.

\subsection{Metodologia}

Para inversão de emulsões conhecem-se dois métodos, o transicional e o catastrófico. No presente estudo utilizar-se-á da inversão catastrófica. Sendo três métodos distintos de preparação de emulsão, um deles de emulsificação direta e dois deles de emulsificação 
dinâmica, nomeados a seguir, nesta ordem: Método I - Adição Água e Óleo concomitantemente antes da agitação; Método II - Adição Gradual de Água em Óleo com Surfactante, durante a agitação; Método III - Adição Gradual de Óleo em Água com Surfactante, durante a agitação.

Como fase oleosa usou-se uma mistura volumétrica de Tolueno PA e n-Heptano PA, e a fase aquosa água destilada. Os surfactantes utilizados foram Tween 80 PS Vetec (tensoativo hidrofílico, HLB 15) e Span 60 Merck (tensoativo lipofílico, HLB 4,7), representando substâncias tais como asfaltenos e ácido naftênico presentes no petróleo, estes foram adicionados a uma concentração mássica de $2 \%$.

Primeiramente, avaliou-se os métodos citados anteriormente. Para obter-se as emulsões, as soluções água-óleo-surfactante foram agitadas no homogeneizador Silverson L4RT a $10000 \mathrm{rpm}$ durante 3 minutos, estas emulsões estavam a $20^{\circ} \mathrm{C}$. Para verificação da inversão utilizou-se o condutivímetro Gehaka CG 1800. A viscosidade das emulsões foi analisada em viscosímetro Brookfiled DV-II+Pro, com esta medida é possível analisar o comportamento reológico ao longo da inversão da emulsão. Usa-se ainda o Microscópio Zeiss Axio Vision onde é possível visualizar o efeito na inversão das emulsões. Equipamentos disponíveis no laboratório Emultec, da UFPR.

Após escolha de um dos métodos, seguiu-se com um planejamento fatorial $2^{3}$ apresentado na Tabela 1 .

Tabela 1 - Planejamento Experimental $2^{3}$

\begin{tabular}{|c|c|c|c|}
\hline Fatores & Nível - & 0 & Nível + \\
\hline Temperatura, ${ }^{\circ} \mathrm{C}$ & 10 & 20 & 30 \\
\hline Composição do Óleo, $\% \mathrm{H} / \% \mathrm{~T}$ & $25 / 75$ & $50 / 50$ & $75 / 25$ \\
\hline Surfactante & Span & $1 / 2$ Span+ $1 / 2$ Tween & Tween \\
\hline
\end{tabular}

\section{RESULTADOS}

Verificou-se nos experimentos realizados que os Métodos I e III se mostram mais interessantes devido à inversão ter acontecido com menor quantidade de água. Pode-se dizer que o método adotado tem influência sobre a inversão das emulsões. Para fins de estudo, escolheu-se o Método I.

Na Tabela 2 são apresentados todos os experimentos que foram realizados. Após a execução dos experimentos, em triplicata, do planejamento experimental da Tabela 1, obtiveram-se os seguintes resultados mostrados na Tabela 3. O Ponto de Inversão é expresso em Porcentagem de água na emulsão, a Redução de Viscosidade em relação a ordens de grandeza reduzidas (comparadas antes e após o ponto de inversão) e estabilidade em horas observadas. 
Tabela 2 - Pontos Experimentais -H: \% de Heptano e T: \% de Tolueno

\begin{tabular}{|c|c|c|c|}
\hline Ensaio & Relação Óleo & Temperatura $\left({ }^{\circ} \mathrm{C}\right)$ & Surfactante \\
\hline 1 & $75 \mathrm{H} / 25 \mathrm{~T}$ & 30 & Tween 80 \\
\hline 2 & $25 \mathrm{H} / 75 \mathrm{~T}$ & 30 & Span 60 \\
\hline 3 & $25 \mathrm{H} / 75 \mathrm{~T}$ & 30 & Tween 80 \\
\hline 4 & $75 \mathrm{H} / 25 \mathrm{~T}$ & 30 & Span 60 \\
\hline 5 & $75 \mathrm{H} / 25 \mathrm{~T}$ & 10 & Tween 80 \\
\hline 6 & $25 \mathrm{H} / 75 \mathrm{~T}$ & 10 & Span 60 \\
\hline 7 & $25 \mathrm{H} / 75 \mathrm{~T}$ & 10 & Tween 80 \\
\hline 8 & $75 \mathrm{H} / 25 \mathrm{~T}$ & 10 & Span 60 \\
\hline $\mathrm{PC}$ & $50 \mathrm{H} / 50 \mathrm{~T}$ & 20 & Span $60+$ Tween 80 \\
\hline
\end{tabular}

Tabela 3 - Resultados do Planejamento experimental $2^{3}$

\begin{tabular}{|c|c|c|c|}
\hline Ensaio & $\begin{array}{c}\text { Ponto de } \\
\text { Inversão }\end{array}$ & $\begin{array}{c}\text { Redução de } \\
\text { Viscosidade }\end{array}$ & Estabilidade \\
\hline 1 & 30 & 2 & Superior a 72 h \\
\hline 2 & 60 & 1 & Superior a 72 h \\
\hline 3 & 30 & 1 & Superior a 72 h \\
\hline 4 & 60 & 1 & Superior a 72 h \\
\hline 5 & 30 & 2 & Superior a 72 h \\
\hline 6 & 90 & 2 & Superior a 72 h \\
\hline 7 & 30 & 1 & Superior a 72 h \\
\hline 8 & 90 & 3 & Superior a 72 h \\
\hline PC & 70 & 1 & Superior a 72 h \\
\hline
\end{tabular}

Os resultados da Tabela 3, ainda estão sendo estudados, mas já se pode afirmar que a temperatura de emulsificação não altera o ponto de inversão, nem a redução na viscosidade quando se utiliza Tween 80, surfactante hidrofílico, tanto em óleos com maior teor de naftênicos, quanto de aromáticos (ensaios 1 e 5, 3 e 7). Segundo Langevin, 2004, emulsões inversas são menos suscetíveis à temperatura. Os melhores pontos (ensaios 1 e 5), considerando \% de água na inversão e redução de viscosidade, foram encontrados nestas condições.

Para o surfactante Span 60, lipofílico, há alteração do ponto de inversão e da redução de viscosidade, nas diferentes composições de óleo e nas temperaturas. Para um mesmo óleo, na temperatura de $30^{\circ} \mathrm{C}$ o ponto de inversão e a redução de viscosidade são menores em relação à de $10^{\circ} \mathrm{C}$ (ensaios 2 e 6,4 e 8 ).

Através do gráfico da Figura 2 a seguir, pode-se observar como é o comportamento da viscosidade ao longo do processo de emulsificação para o ensaio 1. Quando a emulsão aproxima-se do ponto de inversão a emulsão torna-se altamente viscosa, isso se explica devido ao fornecimento de energia cinética às gotículas, segundo Salager (1996). Após esse 
ponto, a viscosidade diminui em duas ordens de grandeza, evidenciando a fase externa aquosa. Em relação ao mesmo ensaio, através da Figura 3, verifica-se como a condutividade da emulsão é alterada durante o processo. Há um pico na inversão de fases, confirmando-se que a fase externa da emulsão agora é a água.

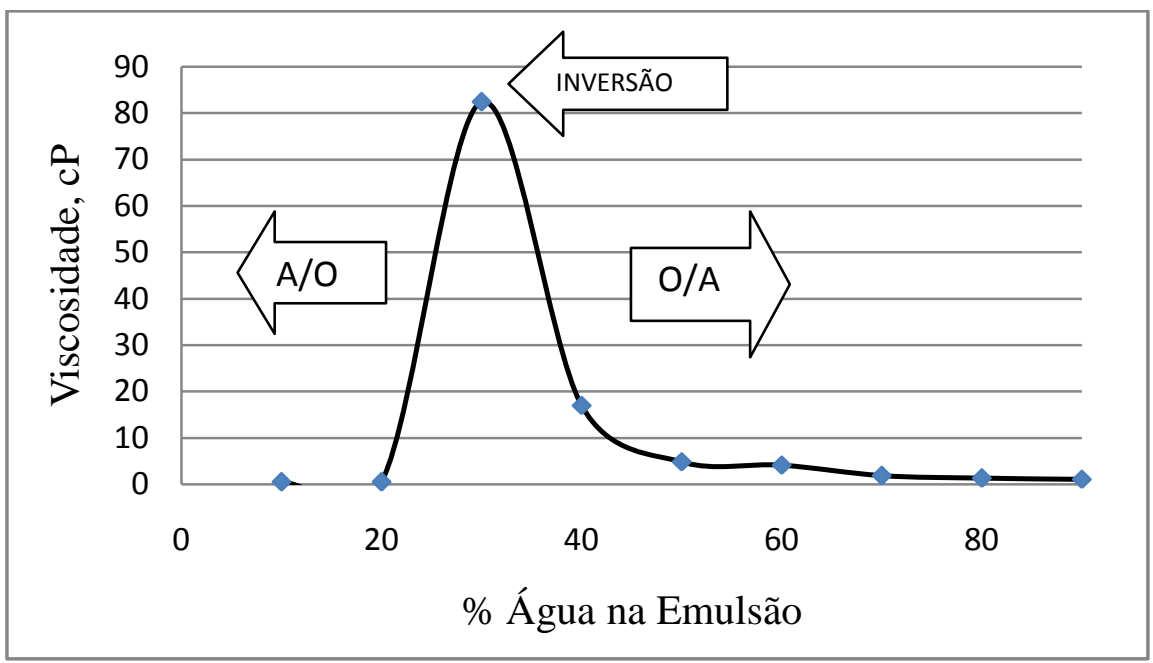

Figura 2 - Comportamento da viscosidade da emulsão, em relação à \% de água - Ensaio 1.

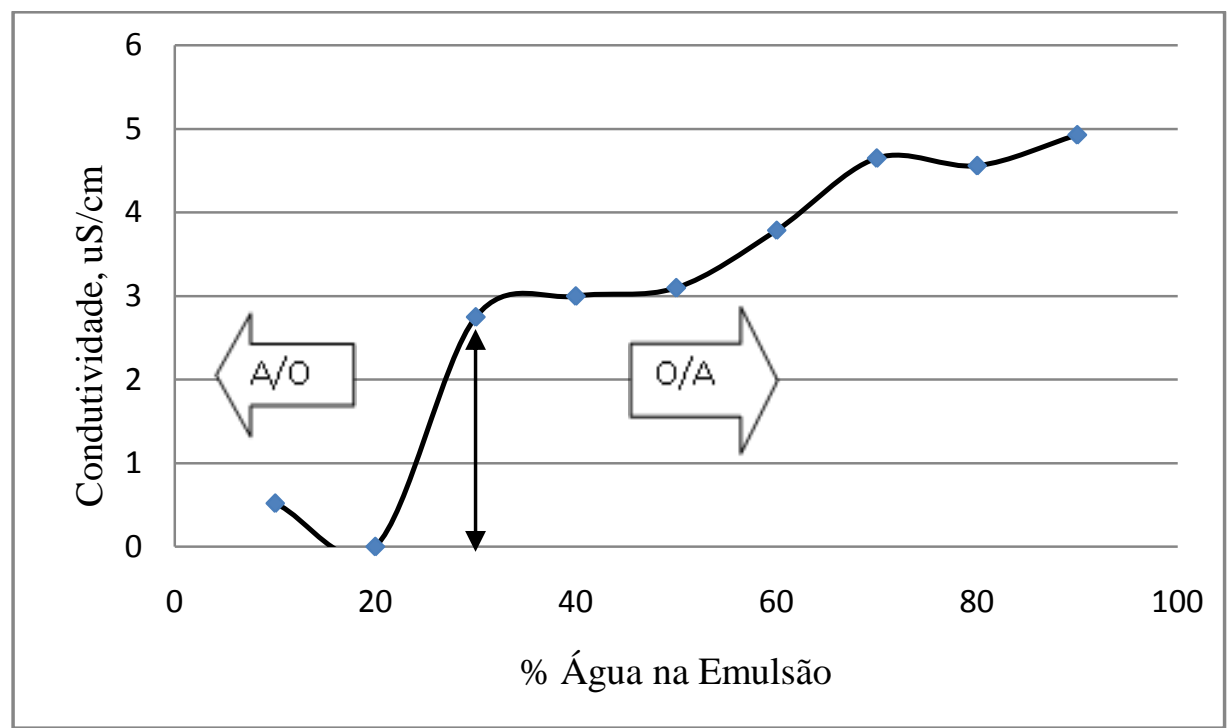

Figura 3. Comportamento da condutividade em relação à \% de água na emulsão - Ensaio 1.

Outra observação a respeito das emulsões é o tamanho das gotas, a seguir nota-se na primeira foto da Figura 4, o ponto da inversão, em que a viscosidade é alta e a condutividade tem um pico de valor. Segundo Silva, 1998, há uma diminuição do diâmetro das gotas próximo ao ponto de inversão, o que aumenta a viscosidade. A redução do tamanho das gotas 
é atribuída ao aumento da viscosidade da emulsão, o que provavelmente auxilia a transferência de energia da agitação para a quebra das gotas (Salager, et al, 1996).
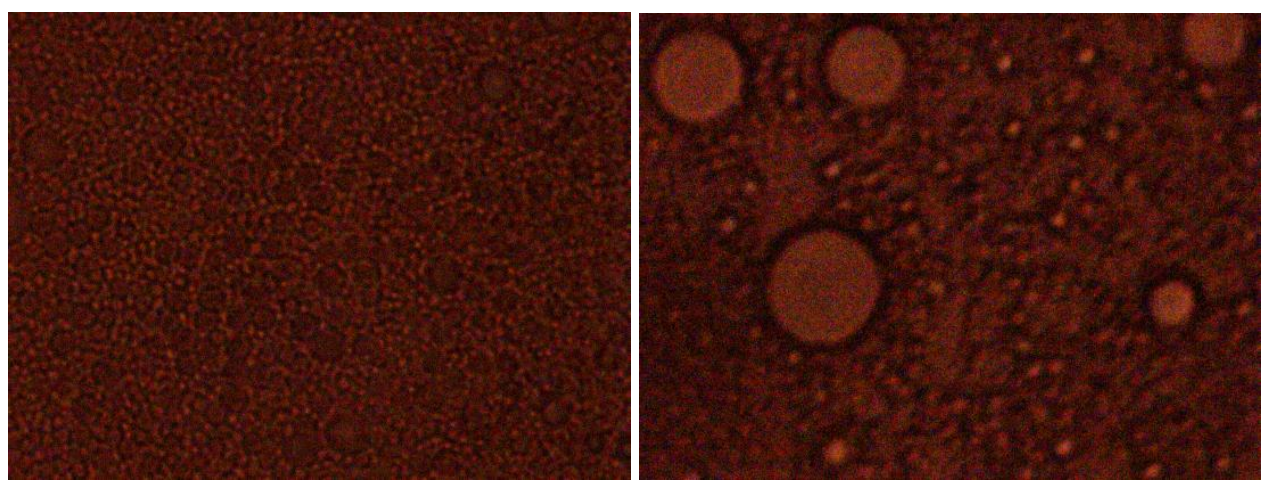

Figura 4. Microscopia das emulsões no ponto de inversão e após, respectivamente - Ensaio 7.

\section{CONCLUSÃO}

Com os resultados obtidos verificou-se a possibilidade de inversão catastrófica de emulsões e que os parâmetros alterados, método de preparação da emulsão, surfactante, temperatura e composição do óleo podem trazer informações importantes no que se diz respeito à porcentagem de água no ponto de inversão e à redução de viscosidade antes e depois deste ponto. Para o surfactante lipofílico utilizado houve alteração do ponto de inversão e da redução de viscosidade, nas diferentes composições de óleo e nas temperaturas estudadas. Já na utilização do surfactante hidrofílico, a temperatura de emulsificação não alterou o ponto de inversão, nem a redução da viscosidade.

Os resultados obtidos permitem avaliar qual a melhor condição de redução da viscosidade, para cada tipo de óleo, por exemplo. Ou ainda outras possibilidades. A redução na viscosidade das emulsões tem alto potencial de aplicação em sistemas de transporte de petróleo, considerando que possuem alta estabilidade, pois seriam estáveis a quebra durante o escoamento. Com a inversão da emulsão de petróleo consegue-se diminuir a perda de carga do sistema, pois a viscosidade da fase externa se torna menor, melhorando assim o escoamento. Desta forma a produção é otimizada, já que se consegue escoar mais petróleo com menor fornecimento de energia, por fim reduz-se os custos na operação, e conseqüente à sociedade brasileira.

\section{AGRADECIMENTOS}

Os autores agradecem ao Programa Interdisciplinar em Engenharia do Petróleo, Gás Natural e Biocombustíveis (PRH-24/UFPR/ANP), ao Laboratório Emultec da UFPR, ao Laboratório de Análises de Combustíveis Automotivos da UFPR (LACAUTets) e ao 
Conselho Nacional de Desenvolvimento Científico e Tecnológico (CNPq) pelo apoio financeiro ao projeto.

\section{REFERÊNCIAS}

BOUCHAMA, F. ;AKEN, G.A. van; AUTIN, A.J.E.;KOPER, G,J.M. On the mechanism of catastrophic phase inversion in emulsions. Colloids and Surfaces A: Physicochemical and Engineering Aspects, v.231, p.11-17, 2003.

BRADLEY, H.B.; Petroleum Engineering Handbook. Third Printing, Society of Petroleum Engineers Richardson, TX, USA, 2001.

DALTIN, Decio. Tensoativos: química, propriedade e aplicações. Editora Blucher. São Paulo, SP, 2012.

LANGEVIN, D.;POTEAU, S.;HÉNAUT, I.;ARGILLIER, J.F. Crude Oil Emulsion Properties and their Application to Heavy Oil Transportation, Oil \& Gas Science and Technology - Rev. IFP, Vol. 59 pp. 511-521, 2004.

SALAGER, J. L. Emulsion Phase Inversion Phenomena. In: SJÖBLOM, J. Emulsion and Emulsion Stability. 2nd ed. Boca Raton: CRC Press, 2006.

SALAGER, J.L.;BRICENO, M.A.;BRANCHO,C.L. Heavy hydrocarbon emulsions making use of the State of-Art in formulation engineering, Encyclopedic Handbook of Emulsion Technology, p. 455-495, 2001.

SALAGER, J.L. Physicochemical parameters influencing the emulsion drop size. School of Chemical Engineering, Merida, Venezuela, 1996.

SCHRAMM, L. L. Emulsions: Fundamentals and applications in the petroleum industry. Washington. American ChemicalSociety, 1992.

SILVA, F.; PEÑA A.;MIÑANA-PEREZ, M.;SALAGER, J.L. Dynamic inversion hysteresis of emulsion containing anionic surfactants. School of Chemical Engineering, Merida, Venezuela, 1998.

SOUZA, Troner Assenheimer de. Inversão de Emulsões de Petróleo usando Partículas Sólidas. Dissertação de Mestrado. Universidade Federal do Paraná, 2009.

THOMAS, Jose Eduardo, Fundamentos de Engenharia do Petróleo. $2^{\mathrm{a}}$ Edição. Rio de Janeiro: Ed. InterciênciaPetrobras, 2004.

TYRODE, E; MIRA, I.; ZAMBRANO, N.; MÁRQUEZ, L.; RONDÓN-GONZALEZ, M.;SALAGER, J.L. Emulsion Catastrophic Inversion from Abnormal to Normal Morphology. Conditions for Triggering the Dynamic Inversion and Application to Industrial Processes.IndEngChem Res. v. 42, p. 4311-4318, 2003. 